\title{
Measuring turbulence in a flotation cell using Electrical Resistance Tomography
}

\author{
Jun Meng ${ }^{{ }^{\star}}$, Weiguo Xie ${ }^{1}$, Kym Runge ${ }^{1,2}$, Dee Bradshaw ${ }^{1}$
}

1. Julius Kruttschnitt Mineral Research Centre, University of Queensland, 40 Isles Rd. Indooroopilly, QLD 4068, Australia

2. Metso Process Technology \& Innovation, Queensland Centre for Advanced Technology, 1

Technology Court, Pullenvale, QLD 4069, Australia

*Jun Meng Email: j.meng2@uq.edu.au

Abstract: Measuring turbulence in an industrial flotation environment has long been problematic due to the opaque, aggressive and abrasive three-phase environment in a flotation cell. One of the promising measurement techniques is Electrical Resistance Tomography (ERT). By measuring the conductivity distribution across a measurement area, ERT has been adopted by many researchers to monitor and investigate many processes involving multiphase flows. In the research outlined in this paper, a compact ERT probe was built and then used to measure the conductivity distribution within a $60 \mathrm{~L}$ flotation cell operated with water and air. Two approaches were then developed to process the ERT data and estimate turbulence related parameters. One is a conductivity variance method and the other is based on the Green-Kubo relations. Both rely and use the fluctuation in the ERT measurement caused by bubbles moving through the measurement area, changing the density of the fluid. Results from both approaches were validated by comparing results produced by the ERT probe in a 60 litre flotation cell operated at different air rates and impeller speeds to that measured using an alternative turbulence measurement device. The second approach is considered superior to the first as the first requires the development of auxiliary information which would not usually be known for a new system.

Keywords: electrical resistance tomography, flotation, turbulence measurement.

\section{Introduction}

Various researchers have highlighted the importance of turbulence controlled processes in flotation cells (Schubert[1] and Fallenius[2]). To characterise turbulence in flotation cells, both CFD modelling and experimental methods can be employed. Some widely used turbulence measurement techniques are: Constant Temperature Anemometer (Sherif[3]), Laser Doppler Anemometry(Sad Chemloul and Benmedjedi[4] ) and Particle Image Velocimetry (Greated, et al.[5] ). All these techniques have their own unique advantages in measuring turbulence in one or two phase turbulent environments. In the case of low concentration of solids, LDA and PIV can also be used to measure turbulence in three phase (solid, liquid, air) flows. However, in an opaque, aggressive and abrasive industrial flotation cell, none of the above techniques can be applied. As a result, the turbulence distributions in the flotation environment are largely based on conjecture or, at best, model simulations. 
Fortunately, there do exist some new measurement techniques that have potential to be applied in the flotation environment, and Electrical Resistance Tomography (ERT) is one of them. ERT gained its first acceptance in 1920s as a geophysical technique for detecting underground structures by converting the electrical measurement data gathered at the surface or from electrodes in boreholes to images. Arrays of electrodes were inserted into the ground. Currents were applied to one pair of electrodes and voltage responses at all others were measured to acquire an image of the underground structure, thereby making it possible to get a more accurate profile of, for instance, oil shales (Dickin and Wang[6]). The basic concept of this technique was further refined by Dines and Lytle (Dines and Lytle[7], Lytle and Dines[8] ), who combined the conventional electrical probing techniques with the more recent tomographic processes used for data reconstruction. Today, with highly efficient data acquisition (DAQ) systems and data inversion software, ERT has been applied to a wide range of scientific and engineering fields such as geophysical studies, clinical diagnosis and chemical or mineral process measurement and optimisation. In theory, any process where the primary continuous phase is to some extent electrically conductive while other elements have different values of resistivity can be investigated and monitored by ERT, and flotation is no exception.

In this research, an ERT probe was built and used to measure the variation in conductivity at localised positions in a 60 litre batch flotation cell operated with water and air. Two different calculation methodologies were developed to process the information produced by the ERT probe to produce a turbulence related parameter. This paper will outline details of the developed ERT probe and data processing procedures as well as the results of experiments performed to validate the ERT measurement results.

\section{Review of the ERT System and its applications}

\subsection{An overview of the ERT system structure and principles of operation}

An ERT system consists of three main components: the electrode sensors, the data acquisition (DAQ) system and the data inversion/image reconstruction software. Fixed at equal distances around the vessel being measured, the electrodes inject current into the fluid and measure the voltage response to enable reconstruction of the variation in resistance across the plane/planes of interest. The purpose of the DAQ system is to do the analogue to digital conversion required to enable communication to and from the data processing computer and the electrodes. There are several data collection strategies, examples include the conducting boundary strategy, the diagonal strategy, the opposite strategy and the adjacent strategy (Dickin and Wang[6]). The adjacent measurement strategy is adopted widely now, and its principle is depicted in Figure 1. 


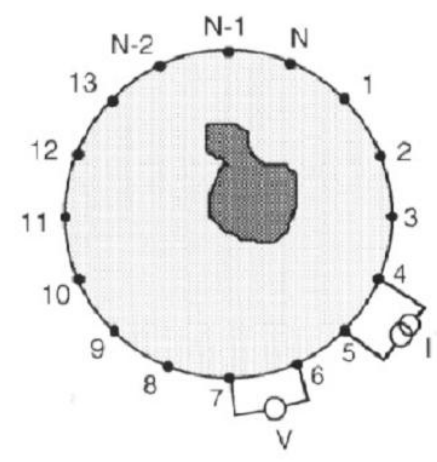

Figure 1: Adjacent measurement strategy (Dickin \& Wang, 1996)

In this strategy, current is applied through 2 adjacent electrodes and the voltage is measured from the neighbouring electrode pairs. Then the next pair of electrodes is applied with current and voltage measurements taken. This process is repeated until all independent measurements have been taken. The total number of independent voltage measurements is $N(N-3) / 2$ (where $N$ is the total number of electrodes) as the current injection electrodes must be excluded from the voltage measurement. $\mathrm{A}$ real-time reconstruction algorithm then uses a regularized and iterative Newton-Raphson algorithm (Hua and Woo[9] ) to calculate the conductivity vector, $\boldsymbol{c}$. The iteration procedure is based on an initial guess of the conductivity distribution updated according to the following equation:

$$
c^{k+1}=c^{k}-\left[f^{\prime}\left(c^{k}\right)^{T} f^{\prime}\left(c^{k}\right)+2 \lambda Q\right]^{-1}\left[f^{\prime}\left(c^{k}\right)\right]^{T}\left[f\left(c^{k}\right)-V\right]
$$

In the above equation, $c^{k}$ is the vector of conductivity values after the $k^{\text {th }}$ iteration, $V$ is the vector of voltages, function $f$ employs the finite element method (FEM) to calculate $f\left(c^{k}\right) ; \lambda$ is a constant which controls the degree of regularization; $Q$ is the regularization matrix and $\mathrm{f}^{\prime}$ is the Jacobian matrix.

The fast computational rates of modern computers provide an opportunity for real time control and display of data. Using a Pentium 2.8GHz 4CPU computer and a mesh of 384 elements and taking into account the mapping time required for the image to be displayed on the screen, a continuous 20 frame per second display rate has been successfully achieved by Wilkinson, et al.[10] .

\subsection{A review of ERT applications in multi-phase systems}

As ERT can be used as a non-intrusive technique to measure conductivity distribution in a crosssection of a disturbed fluid, with real time imaging and high accuracy, it can be applied in the study of multi-phase systems. Dong, et al.[11] presented a 16 channel ERT system with a fully programmable and reconfigurable FPGA (field programmable gate array) and Compact $\mathrm{PCl}$ (peripheral component interconnect) bus, which was shown to have a high frame rate, signal-to-noise ratio and low error associated with repeat measurements. This has provided a powerful platform for measurement in multiphase flows. Cilliers and his co-workers have developed and applied an ERT system (Wang and Cilliers[12],Cilliers, et al.[13] ) to study foams and in particular to measure the foam density distribution. 
Cilliers and his co-workers also tested ERT in solid - liquid systems (Stevenson, et al.[14] ,Harrison, et al.[15] ). Jin, et al.[16] studied the Sauter mean bubble size and rise velocity of air bubble swarms in a laboratory scale bubble column using ERT. Based on the differences in rise velocity of a bubble swarm across a cross-section monitored by ERT, gas holdups and the bubble size distribution of both large bubble swarms and the small ones could be obtained. Abdullah, et al.[17] analysed the hydrodynamic attributes of a three phase (gas, solid, liquid) mixture in a stirred vessel with ERT to obtain the dispersed phase-holdup distribution as a function of impeller Reynolds Number and solid particle loading, by using the fact that holdup $\varepsilon_{\mathrm{d}}$ can be calculated using electrical conductivity values:

$$
\varepsilon_{d}=\frac{2 \sigma_{1}+\sigma_{2}-2 \sigma_{m}-\sigma_{m}\left(\frac{\sigma_{2}}{\sigma_{1}}\right)}{\sigma_{m}-\frac{\sigma_{2}}{\sigma_{1}}+2\left(\sigma_{1}-\sigma_{2}\right)}
$$

Where $\sigma_{1}$ is the continuous phase (water) conductivity, $\sigma_{2}$ is the dispersed phase conductivity and $\sigma_{m}$ is the conductivity of the mixture measured by ERT. Kourunen, et al.[18] used a 3D ERT in a 50L mechanical flotation cell to measure gas holdup distribution as a function of rotor speed, air flow rate and impeller-stator design. Heikkinen, et al.[19] proposed an Electrical Impedance Tomography (EIT) approach with efficient current injection and voltage measurement protocols for measurement, reconstruction and visualization of 3D images with a frame rate of 15 , based on which the 3D air/liquid distribution in a stirred vessel was visualised in real time and material flow could be monitored.

Liter, et al.[20] developed a novel EIT diagnostic system and applied it to an electrically conducting slurry bubble column reactor to quantitatively measure material distribution in both two phase and three phase flows over a range of column pressures, superficial gas flow rates and two different solid loadings. The results were validated against a gamma-densitometry tomography (GDT) diagnostic system and revealed a maximum difference of 0.15 volume fraction.

Xie, et al.[21] employed an ERT system with a switched bi-directional constant current source to measure voltages across the foam and estimated the average bubble size of the flowing foam in a foam column from acquired voltage data. The result was compared with directly measured data using a digital camera and agreement was found. Xie, et al.[22] used a similar ERT system to identify the coarse foam regions and foam coalescence areas in a flowing foam column. Meng, et al.[23] combined a 16 electrode ERT system with a Venturi meter to measure the flowrate of a two-phase water-liquid flow. The conductivity data provided by the ERT were processed by a least square support vector machine (LS-SVM) classification method to identify the flow pattern and calculate the void fraction and mass quality. These values were then combined with the differential pressure measured by the Venturi meter to calculate the mass flowrate. As the ERT system did not have to display images, fast and real time measurements were achieved.

Lehikoinen, et al.[24] imaged industrial scale flotation cells using an intrusive probe sensor linked to a 3-D ERT system and found the sensor could be used to measure stiffness of the froth as well as indicate the position of the froth-pulp interface. Also the automatic calibration technique employed 
could eliminate the contamination effects caused by the invasive measurements. Kourunen, et al.[25] measured the spatial distribution of gas holdup at various impeller speeds and gas flow rates in a 50 litre flotation cell using an electrical tomography imaging technique. In other research, Kourunen, et al.[26] estimated the 3D gas holdup distribution in a mechanical flotation cell under different conditions with a three dimensional ERT device.

Bolton and Primrose[27] reviewed several applications of ERT in pharmaceutical and related areas, including measurement of solid distribution in stirred tanks, monitoring of an industrial pressure filter and flow profile and velocity measurement in a physical model of a catalytic reactor.

These applications clearly demonstrate that ERT is capable of measurement in multiphase systems. However, no work to date has been performed to apply ERT as a tool for measuring turbulence in flotation. This is the objective of the work presented in this paper.

\section{Experimental design}

\subsection{Details of the ERT device developed in this study}

Due to the large dimensions of a typical industrial flotation cell, it would be difficult and impractical to place ERT electrodes around its outer shell. In addition, measurement of turbulence is best performed at a localised point - due to its large variation throughout the volume of the flotation machine. Therefore, a small intrusive probe capable of placement at a particular position in a tank is more suitable for turbulence measurement in flotation cells. An ERT sensor based on this principle was made by fixing 16 electrodes equally distanced on a plastic plate, which was in turn fixed to a metal circular truncated cone with a long iron tube at the end through which wires could be passed through and housed. The measurement plane was then defined by the circular plane surrounded by the 16 electrodes. The probe was connected to an ERT box provided by the University of Cape Town (UCT), which acted as the data acquisition device. Data were collected by the UCT ERT using the adjacent measurement strategy described in Figure 1. These data were then transferred to a computer where the UCT Tomography software did the data inversion and image reconstruction process based on the single step iteration algorithm outlined in Equation (1). The probe and the UCT ERT DAQ box and computer for data processing are depicted in Figure 2. 


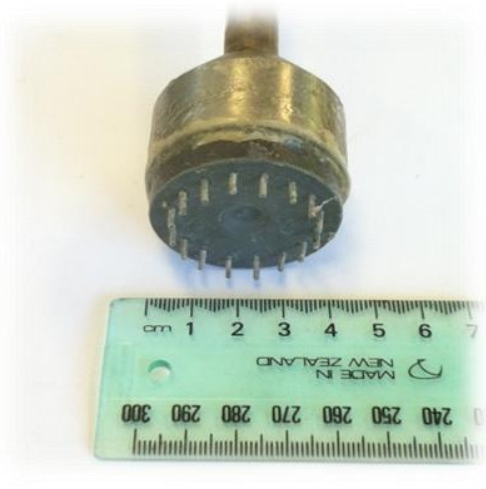

(a) ERT probe

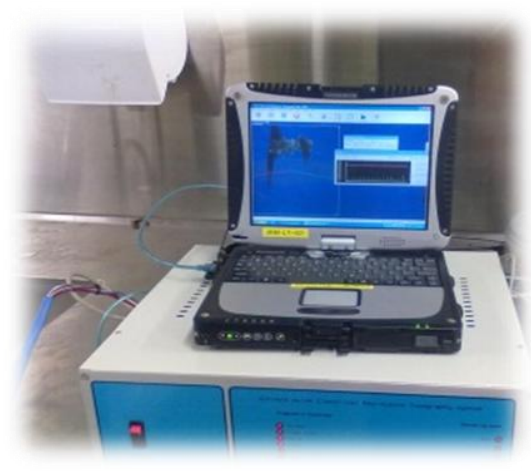

(b) UCT ERT Device

Figure 2: Components of the ERT sensor system

The ERT measures the conductivity distribution across the circular plane defined by the electrodes. A finite element method employed by the UCT Tomography software splits the measurement area into many small elements for which conductivity values can be calculated using the measured voltage data. UCT Tomography software is based on open source code, EIDORS (Electrical Impedance Tomography and Diffuse Optical Tomography Reconstruction Software, http://eidors3d.sourceforge.net/). The Image reconstruction algorithm in EIDORS was developed by Polydorides[28] .

\subsection{Experimental Details}

Measurements were made of turbulence using the ERT probe in a 60 litre batch flotation cell, as depicted in Figure 3(a). This cell utilises an Outotec style impeller/diffuser system. For ERT to be able to determine the turbulence distribution in the cell, air bubbles must be present in the system. Movement of air bubbles across the cross-section of the probe will result in variation in the conductivity measured. The variation in conductivity with time is hoped to be correlated with the turbulence at the point of measurement. Experiments were performed at a range of different gas rates and impeller speeds. In addition, frother was added to the flotation cell to make the bubbles more evenly distributed in size. The frother used was MIBC, the concentration was 33ppm (2ml in 60 litres of water).

Measurements were performed along a chosen vertical axis (see Figure $3(b)(c)$ ). On this vertical axis, a series of measurement positions were chosen, namely $-98,-93,-83,-73,-63,-53,-43,-33,-23,-13$, $3,7,17,27,47,67,87,117$ and $147 \mathrm{~mm}$ relative to the stator plane. 


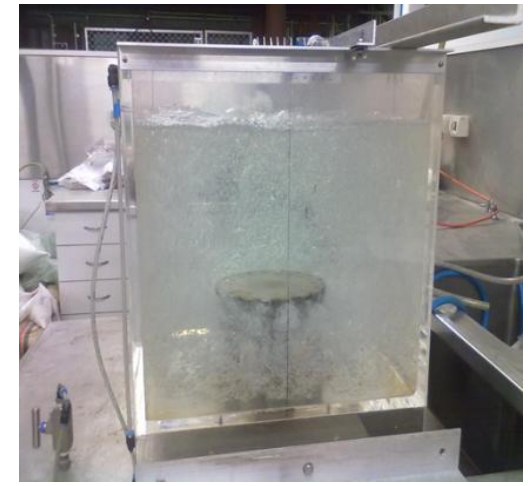

(a) 60 litres batch flotation cell with air addition

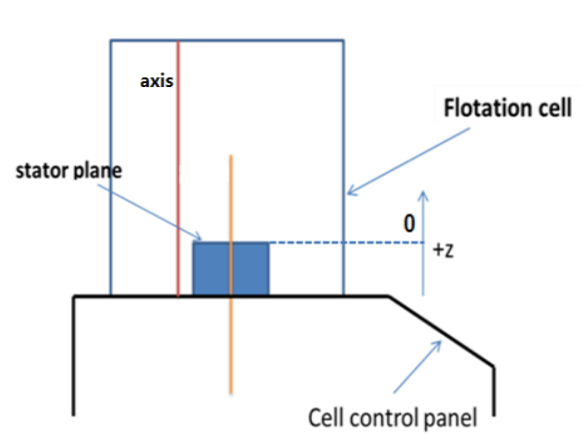

(b) ERT measurement axis side view

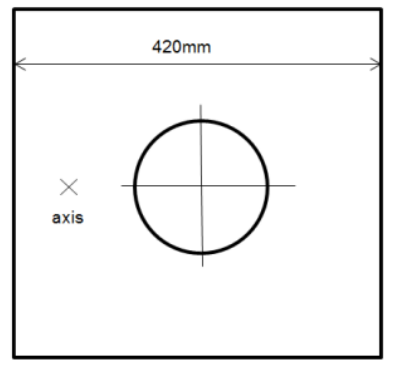

(c ) ERT measurement axis plan view

Figure 3: Experimental details

At each position, 7500 frames of data were recorded for later off-line analysis. Prior to the voltage data being collected, however, a calibration at the point of measurement was performed in which voltage measurements were collected without application of a current. This is because different positions in the cell may have a different influence on the distribution of the electrical field produced by the ERT probe, due to the influence of geometrical factors such as the impeller and stator. The calibration data recorded for each measurement position were later used in the calculation of the voltage and conductivity values.

To validate the ERT probe measurement results, they need to be compared to another technique capable of measuring turbulence in the flotation cell. However, since the ERT measurement can only be performed when air is present in the fluid, it needs a technique capable of turbulence measurement in a multiphase system. Recently, a Piezoelectric Vibration Sensor (PVS) has been developed and demonstrated to be capable of measuring turbulence in this environment(Meng, et al.[29] ,Tabosa, et al.[30] ). The sensor consists of a piezoelectric film which when deflected backwards and forwards produces an AC current. Meng, et al.[29] has developed techniques for processing this information and has shown it can be used to provide an estimation of the fluctuation in kinetic energy at the point of measurement. Measurement can be performed, reliably, in the presence of bubbles and/or high concentrations of solids. Therefore it provides a good baseline for comparison of the ERT measurement results. The PVS measurement was therefore also performed in the $60 \mathrm{~L}$ cell in Figure 3 at the same measurement positions as used for the ERT probe.

Although the size of the ERT probe to the size of the flotation cell is relatively large which may lead to interference to the flow field in the cell, the major objective of this work is to evaluate the ability of the ERT probe to measure turbulence in multiphase environments by comparing it to PVS results. Once this has been demonstrated, the ERT probe will usually be applied in much bigger cells where the interference of the intrusive probe can be ignored. 


\section{Methodologies for processing the ERT results to estimate turbulence}

Turbulent flow consists of many rotating eddies of varying size and velocity, resulting in fluctuating fluid velocities. To describe turbulence in a flotation cell, a number of quantities may be needed (Mathieu and Scott[31]). Assuming fluid velocity is $V_{i}$ in a certain direction, the following turbulent related parameters can be defined:

- $\bar{V}_{i}$,time averaged flow velocity in a specific direction

- $v_{i}=V_{i}-\bar{V}_{l}$, fluctuating velocity in a specific direction

- $v_{i}^{\prime}=\sigma_{v_{i}}$, velocity fluctuation, a statistical value representing the fluctuating velocity, measured as the standard deviation of $v_{i}$

- $\quad$ TKE $=\frac{1}{2}\left(v_{1}^{\prime 2}+v_{2}^{\prime 2}+v_{3}^{\prime 2}\right)$, turbulence kinetic energy (TKE). TKE is calculated from the root mean square of fluctuations of the turbulent fluid velocities in all directions.

- $\sigma_{v_{i}^{2}}$, kinetic energy fluctuation, measured as the standard deviation of $v_{i}^{2}$

The purpose of this study was to develop a methodology to relate what is measured by the ERT probe to a turbulence related parameter. It was decided to take advantage of the fact that there will be variation in the conductivity measured between the electrodes of the ERT probe when placed in a flotation cell as a consequence of bubbles moving through the measurement area, changing the density of the system, at a fluctuating velocity.

Two methods were developed and tested for obtaining the turbulence information using ERT measurement: a conductivity variance method and a Green-Kubo method, the details of them are described below.

\subsection{Conductivity variance method}

The first approach involves developing the expected relationship between the variance in the measured conductivity and fluid velocity parameters. This is achieved through a series of assumptions. In a multiphase turbulent flow, the conductivity (C) measured at a certain position by ERT can be formulated as:

$$
C=f(\rho(v))
$$

Where, $\rho$ is density of the multiphase fluid, and $v$ is the velocity of the flow at the measurement position. Therefore, the variance of conductivity can be expressed as:

$$
\sigma_{C}^{2}=\left(\frac{\partial f}{\partial \rho}\right)^{2}\left(\frac{\partial \rho}{\partial v}\right)^{2} \sigma_{v}^{2}
$$

In a flow with air bubbles, conductivity $C$ increases with density of the fluid monotonously(Weaire and Hutzler[32] [39]). In a narrow range, $C$ can be considered as proportional to density $\rho$. Therefore, $\frac{\partial f}{\partial \rho}$ is 
a constant. To derive $\frac{\partial \rho}{\partial v}$, bubble movements in the flotation cell have to be considered. From a theoretical analysis of the flotation system, the following equation was proposed:

$$
\frac{\partial \rho}{\partial v}=\frac{\left(\rho_{\text {water }}-\rho_{\text {air }}\right) V_{\text {air }}}{A v^{2}}
$$

Where $V_{\text {air }}$ is the air flow rate, i.e. the volume of air pumped into the cell in unit time, $v$ is bubble velocity, $A$ is the cross sectional area of the cell. Combining (5) and (6)

$$
\sigma_{c} \propto \frac{\sigma_{v}}{v^{2}}
$$

Since from error propagation theory(Fornasini[33] ), $\sigma_{v^{2}}^{2}=4 v^{2} \sigma_{v}^{2}$, therefore

$$
\sigma_{c} \propto \frac{\sigma_{v}^{3}}{\sigma_{v^{2}}^{2}}
$$

At each measurement position, the measurement plane can be divided into many elements. The $C$ value of each element can be reconstructed from the raw voltage measurement data using a finite element method (FEM). The UCT ERT device can capture approximately 793 frames of voltage data in one second. In each frame, there are 16 groups of voltage measurements, in which every group is composed of 13 measurements of voltage at consecutive electrode pairs starting from and ending at the adjacent of, but excluding, the current injection electrodes, resulting in 208 voltage values for one frame. For the 7500 frames acquired at each measurement position in the 60 litre flotation cell (as outlined in Section 3.2), every 30 frames of data were averaged and recorded by the ERT DAQ box to get 250 frames of measurement data, rendering a frame rate of approximately 25 frames per second. These frames can be grouped into 5 sets, with about 50 frames in each set, to derive the standard deviation of the measurement values. The calculated standard deviation of conductivity measured in this work was found to be insensitive to how many frames of data were averaged. Figure 4 compares the standard deviation calculated at different positions along a vertical axis $150 \mathrm{~mm}$ from the centre when the cell was operated at an impeller speed of $370 \mathrm{rpm}$ and air flow rate $40 \mathrm{~L} / \mathrm{min}$ when the data block sizes were 10 and 30 frames, respectively.

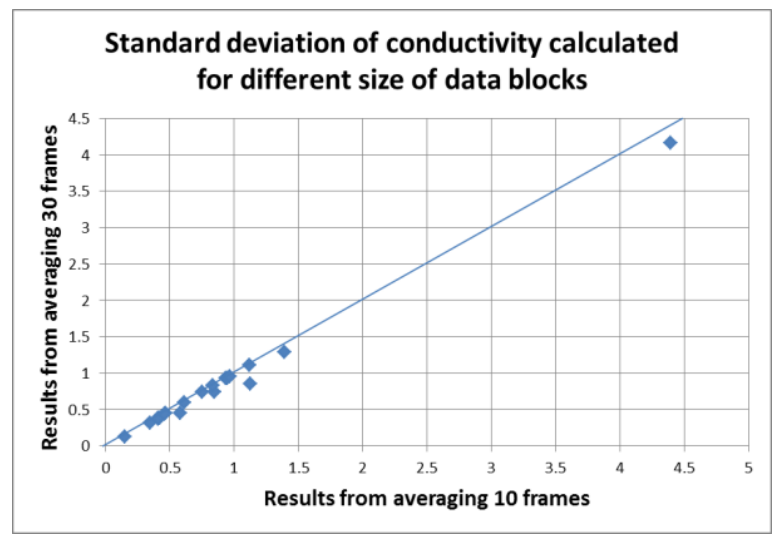


Figure $4 \mathrm{~A}$ comparison of standard deviation calculated at different positions in a flotation cell when 10 frames were averaged compared to 30 frames

The data file for each measurement position was later used for off-line data reconstruction and conductivity calculation, which was performed using Matlab code provided by UCT. Since the conductivity values reconstructed from the raw voltage data were relative, not absolute, values, standard deviation values of the conductivity were used. A mesh of 836 elements for each frame was selected in the reconstruction process and the average value of standard deviation of capacitance $\sigma_{c}$ at each measurement position was calculated by averaging the standard deviation of each element. It should be noted that there was uncertainty associated with the reconstructed conductivity values which originates from both the measured raw voltage data and the image reconstruction algorithm. Because of the calibration process, effects from mismatched amplifier gains and electrode position errors can largely be erased and only $1 \%$ of variations in symmetry between each cycle of the FEMpredicted waveform exist, which are determined by the layout of the FEM mesh with respect to the electrodes (Wilkinson, et al.[10] ). Therefore, the uncertainty associated with the voltage data mainly comes from the quantization noise and the system thermal noise, which are normally negligible. The reconstruction algorithm used in this method was designed for real time display of the conductivity map, which needs to be computationally efficient, and therefore only the one step Newton-Raphson iteration was used to update the initial guess of the conductivity distribution. Such an approach will bring approximation errors which can be compensated for by representing the error as a noise process in the measurement model (Nissinen[34]). However, this was considered beyond the scope of the data processing reported in this paper. It has been found that the one step Newton-Raphson iteration is accurate enough for visualization (Wilkinson, et al.[10] ) and therefore can be used as a rough representation of the real conductivity distribution.

From equation (8), the correlation between conductivity fluctuation $\sigma_{c}$, velocity fluctuation $\sigma_{v}$ and fluid kinetic energy fluctuation $\sigma_{v^{2}}$ can be established. This correlation demonstrates that variation in conductivity measured by the ERT probe can be related to turbulence related parameters of the fluid flow $\left(\sigma_{\mathrm{v}^{2}}\right.$ and $\left.\sigma_{v}\right)$ It is not straightforward, however, because $\sigma_{\mathrm{v}^{2}}$ or $\sigma_{v}$ cannot be obtained independently of each other.

To validate Equation (8) and show that ERT is capable of measuring turbulence related parameters, it was decided to compare ERT results with the PVS outlined in Section 3.2. PVS is a sensor that has been shown to be capable of measuring kinetic energy fluctuation $\left(\sigma_{v^{2}}\right)$ in the flotation environment where air bubbles are present(Meng, et al.[29] ). Therefore, a method is required to calculate $\sigma_{v^{2}}$ from $\sigma_{c}$, which will be measured by ERT. From LDA turbulence measurement data performed by Brennan[35] [41] in the same 60 litre batch flotation cell as described in Section 3.2 , it can been derived that 


$$
\sigma_{v^{2}} \propto \sigma_{v}^{2}
$$

A trial of the direct substitution of $\sigma_{v} \propto \sqrt{\sigma_{v^{2}}}$ from Equation (9) into Equation (8) failed to result in a correct prediction when ERT and PVS data were directly compared. This was found to be a consequence of the fact that $\sigma_{v^{2}}=2 v \sigma_{v}$, where $\sigma_{v^{2}}, \sigma_{v}$ and $v$ are all functions of impeller speed (IS) and position within the flotation cell. Therefore, a correction for velocity which will be a function of IS and cell position has to be introduced into the calculation. To establish this correction for a water only system, PVS measurements of $\sigma_{v^{2}}$ were carried out along a vertical axis $123 \mathrm{~mm}$ from the centre of the cell at nine different impeller speeds. The correlation between $\sigma_{v^{2}}$, impeller speed IS and measurement position is plotted in Figure 5(a) and 5(b). Each symbol in the graphs represents a different measurement position (relative height to stator plane). The abscissa is impeller speed IS and the ordinate is kinetic energy fluctuation $\sigma_{v^{2}}$.

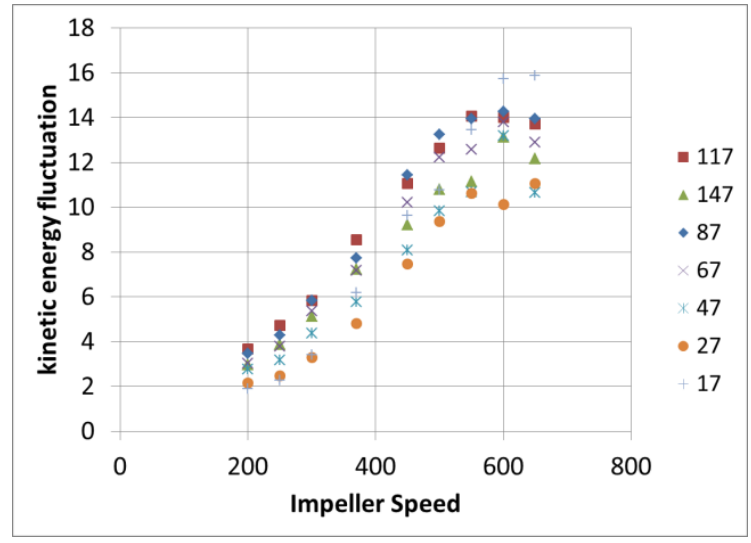

(a) Kinetic energy fluctuation in low turbulence zone

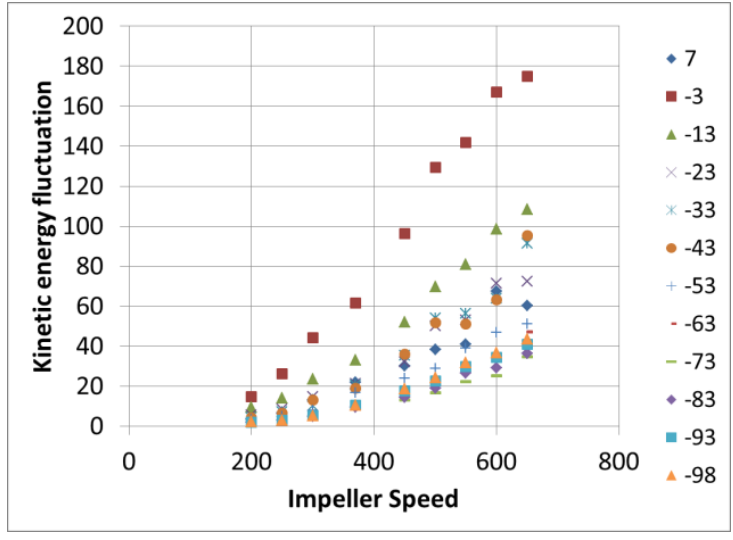

(b) Kinetic energy fluctuation in high turbulent zone

Figure 5: Kinetic energy fluctuation as a function of impeller speed measured at different heights in a flotation cell (relative to the stator plane)

From Figure 5 it can be seen that kinetic energy fluctuation varies with both impeller speed and measurement position. A model is therefore needed to correlate kinetic energy fluctuation with both of these variables. A measurement radius was defined as $R=\sqrt{d^{2}+h^{2}}$, where $d$ was the horizontal distance from the centre of the cell to the measurement position and $h$ was the height of the measurement position relative to the bottom of the cell. Then a normalized measurement radius was defined as $r=R / D$, where $D=420 \mathrm{~mm}$ was the cell's dimension. By assuming $\mathrm{N}$ is the number of times the impeller rotates in one minute (dimensionless), the normalized kinetic energy fluctuation $S_{v^{2}}=$ $\sigma_{v^{2}} / \max \left(\sigma_{v^{2}}\right)$ is the ratio between the measured kinetic energy fluctuation to the maximum kinetic energy fluctuation measured by the piezoelectric sensor in all the experiments. Then it can be modelled that: 


$$
S_{v^{2}}=a(r) N^{b(r)}
$$

The corresponding $a$ and $b$ values at each measurement height were derived from LSM curve fitting of the different impeller speed data. Then fitting $a$ and $b$ values to $r$ yield:

$$
\begin{aligned}
& \mathrm{a}=6.735 \times 10^{-5} e^{-\left(\frac{r-0.5846}{0.1039}\right)^{2}} \quad\left(R^{2}=0.9831\right) \\
& \mathrm{b}=15.36 r^{2}-18.66 r+6.754\left(\mathrm{R}^{2}=0.9232\right)
\end{aligned}
$$

Figure 6 depicts the fitted curves from equations (11) and (12) versus the measurement results.
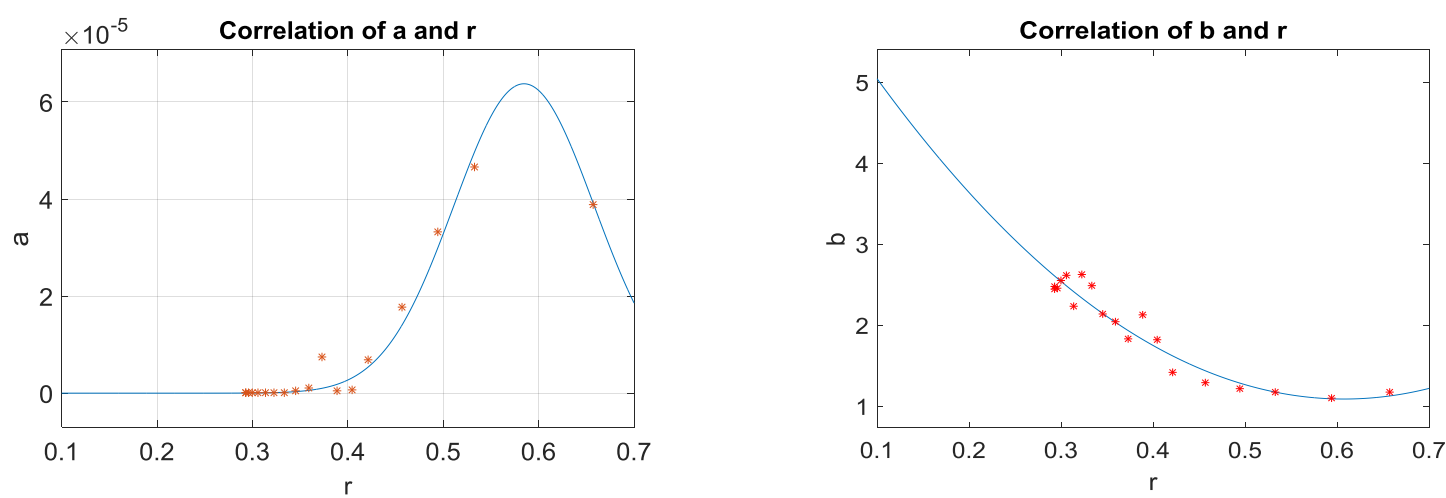

Figure 6 Calculated $a$ and $b$ parameter values versus the measured results

By combining equation (9) and (10), the following is obtained:

$$
\sigma_{v}^{2} \propto a(r) N^{b(r)}
$$

Next by combining equation (8) and (13), the kinetic energy fluctuation can be calculated as:

$$
\sigma_{v^{2}} \propto \frac{a(r)^{0.75} N^{0.75 b(r)}}{\sqrt{\sigma_{c}}}
$$

It should be noted that equations (13) and (14) are proportional correlations; therefore they don't have to be dimensionally balanced. The imbalance in dimensions can be compensated for by dimensional coefficients which are not currently shown in the equations.

Equation (12) was developed using LDA and PVS measurements performed in a water only system. It is assumed that it will also apply for multiphase flows where air and slurry particles are introduced to the flotation system. To test this hypothesis, the kinetic energy fluctuation estimated using ERT in a flotation cell operated with water and air bubbles will be compared to the results produced by PVS in Section 5.1 . 


\subsection{Method based on the Green-Kubo relations}

The second approach explored for converting the ERT measurement to a turbulence related parameter was based on the Green-Kubo relations(Green[36] ,Kubo[37] ),regardless of whether transport processes are thermally or mechanically stimulated, the field created (be it thermal, electric, or magnetic) will have a flux that will be linearly related to the applied force. This can be expressed as a linear constitutive relationship:

$$
J=L\left(F_{e}=0\right) F_{e}
$$

Where $J$ is the thermodynamic (or electric, or magnetic field) flux, $L(0)$ is called a linear transport coefficient and $F_{e}$ is a fluid force. The calculation of $L(0)$ is expressed as:

$$
\left.L(0)=\beta V \int_{0}^{\infty} d t J(t) J(0)\right\rangle=\sigma_{J}^{2} \beta V \int_{0}^{\infty} c_{J}^{(t)}(0, t) d t
$$

where $\beta=\frac{1}{k T}$, and $V$ is the system volume. Operator $\langle\bullet\rangle$ is the calculation of auto-covariance, $c_{J}^{(t)}(0, t)$ is the autocorrelation coefficient for the flux at time 0 and $\mathrm{t}, c_{J}^{(t)}(0, t)=\frac{\overline{J(0) J(t)}}{\sigma_{J}^{2}}$.

When applied to the flotation systems being measured by the ERT, $J$ is the electrical flux $\Phi_{E}$ which can be calculated from the ERT data and $L(0)$ can be approximated by calculating the sum of several temporal correlation coefficients of consecutive ERT data frames. The electrical flux is defined as the number of electrical field lines passing through a given area and can be formulated as $\Phi_{E}=\iint_{S} E \cdot d S$, where $E$ is the electrical field magnitude, $S$ is the surface of the considered area over which the integral is performed. Since electrical potential is defined as $U=E / d$, where $d$ is the distance between the two measurement points, the voltage measured at the ERT electrodes can therefore be considered as a proportional representation of $E$ between the two electrodes as the distance between electrodes is constant. By adding up all the voltage values in a frame measured by ERT, the value of $\Phi_{E}$ in the time span of a frame can be approximated proportionally. For calculation of $L(0), 8$ consecutive ERT frames were chosen and temporal correlation coefficients between frame one and every following frames were calculated separately. By adding up these coefficients, $L(0)$ was approximated. For frames after the eighth frame, they were considered uncorrelated to the first frame because the time span between any one of them and the first frame was sufficiently large. It should be noted that since this calculation process involves calculating correlation coefficients between adjacent frames, the frame rate was set to a maximum (approximately 793 frames per second without averaging frames) to make sure the time span between adjacent frames was small enough to capture the localised variation caused by the turbulence.

Next by calculating $\Phi_{E} / L(0), F_{e}$ can be determined. To correlate $F_{e}$ with turbulent velocity, it is assumed that the fluid mass passing through the measurement plane in a short period of time $\Delta t$ (time interval between two adjacent ERT data frames) is a fixed value, $m$. By applying the Impulse- 
Momentum Theorem, $=F_{e} \Delta t=m \Delta v$, then the velocity of the fluid can be expressed as $\Delta v=v_{t 2}$ $v_{t 1}=\frac{F_{e} \Delta t}{m} \propto F_{e} \Delta t=\Delta I$. Since $\sigma_{v_{t 2}}=\sigma_{v_{t 1}}=\sigma_{v}$, therefore, $\sigma_{\Delta v} \propto \sigma_{v} \propto \sigma_{I}$.

Thus from the above analysis it is hypothesised that the fluid impulse fluctuation $\left(\sigma_{I}\right)$ that can be estimated from the ERT probe conductivity measurements should be proportional to the velocity fluctuation of the fluid across the measurement plane. In Section 5.2, the calculation results were compared with PVS measurement results to test this hypothesis.

In the calculation of $\Phi_{E}$ and $L(0)$, only the voltage measurement data were used. Therefore, there was no error as a consequence of the image reconstruction algorithm as it was not used in the calculation. The error in the voltage measurement could largely be negated by applying the calibration algorithm reported by Wilkinson, et al.[10], resulting in the irremovable quantization noise and system thermal noise being the main source of errors.

\section{Results of Validation of the ERT Measurement Methodologies}

\subsection{Validation of the conductivity variance method of predicting fluid kinetic energy fluctuation}

According to Equation (14), kinetic energy fluctuation can be estimated by measuring the variation in the conductivity distribution using the ERT probe and correcting this value based on a modelled effect of impeller speed and the position of measurement. The validity of this calculation was assessed by comparing the predicted $\sigma_{v^{2}}$ values calculated using the ERT measurement data and the piezoelectric sensor measurement data performed in the 60 litre batch flotation cell described in Section 3.2. The measurement was carried out along a vertical axis $123 \mathrm{~mm}$ from the cell's centre at different impeller speeds, namely $370,450,550$ and $650 \mathrm{rpm}$. The values of $\sigma_{c}$ were obtained by performing five measurements using the ERT probe. Air rate was kept constant at $10 \mathrm{~L} / \mathrm{min}$ and MIBC concentration was 33ppm. For each of the measured $\sigma_{\mathrm{c}}$ values, a $\sigma_{\mathrm{v}^{2}}$ value was calculated by using the model defined by equations (11), (12) and (14). To enable comparison, piezoelectric sensor readings were collected at these same positions whilst the cell was operated at the same set of operating conditions. Turbulence profiles obtained by plotting the results of these two measurement techniques are shown in Figure $7(\mathrm{a})$ and $7(\mathrm{~b})$. It can be seen that the turbulence profiles drawn with the two techniques are quite similar. When data from the two measurement techniques are plotted against one another on the same graph, a linear correlation can be observed, as is shown in Figure 7(c). 


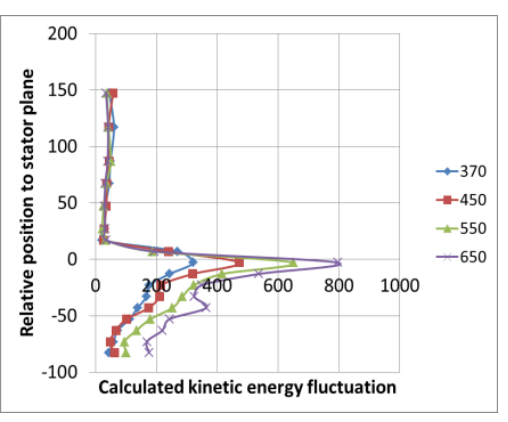

(a) ERT calculated kinetic energy fluctuation

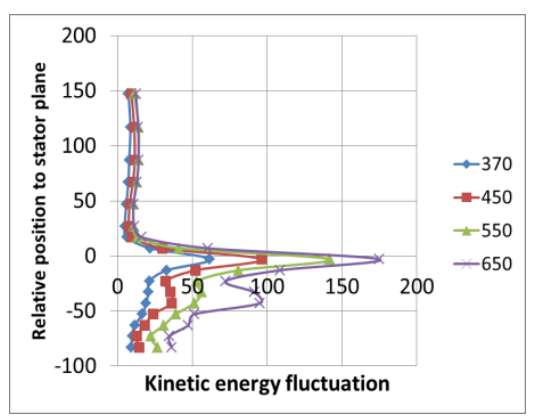

(b) PVS measured kinetic energy fluctuation

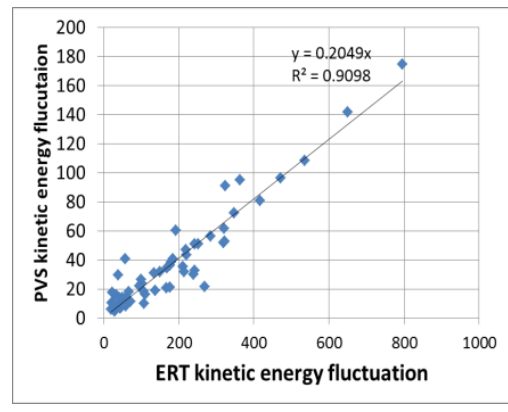

( C) Correlation between PVS and ERT

Figure 7: Turbulence profiles measured using ERT and the piezoelectric sensor

These results validate the assumptions underpinning equation (14). These results are proof that variation in the conductivity measured by ERT can be correlated with the intensity of the turbulence of the fluid. However, as the model correction for velocity in Equation (14) was derived from data performed in a water only system, velocities would be expected to deviate in a cell when air is present within the system. The greater the amount of air, the greater the expected deviation. To validate the model in a broader sense, ERT and PVS measurements were taken along an axis $150 \mathrm{~mm}$ from the centre at a higher air rate. The ERT DAQ box was set to record all 7500 frames for each measurement position without averaging every 30 frames. This configuration was used so as to facilitate the Green-Kubo analysis discussed in section 4.2. The averaging was done offline using MATLAB code. Figure 8 shows the PVS results and ERT results measured along the axis $150 \mathrm{~mm}$ from the centre when higher air rates were used (40,80 and $100 \mathrm{~L} / \mathrm{min})$. A comparison between the PVS and the ERT measurements was also plotted. It can be seen that comparison between the PVS and ERT measurements becomes poorer as the air flow rates increase. It is concluded that this conductivity variance method cannot be used when the air flow rate is high. In addition, the conductivity method was established by modelling kinetic energy fluctuation from the measurement data obtained by the piezoelectric sensor, making this method dependent on another measurement technique, and equations (11) and (12) may not fit very well for measurement positions other than along the $123 \mathrm{~mm}$ axis. Therefore the models developed in this system cannot be transferred to other systems.
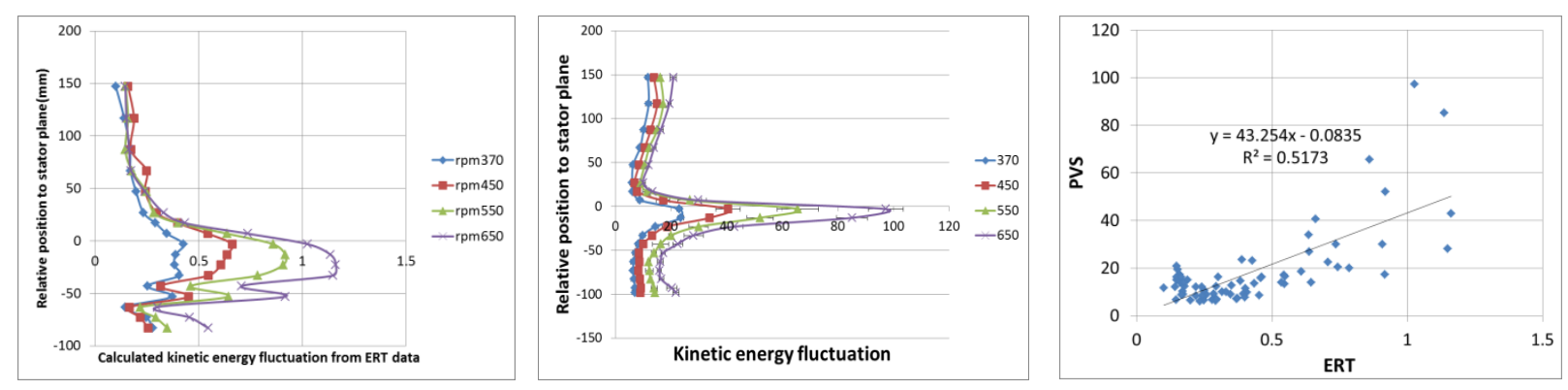
(a-1) ERT result, air rate $40 \mathrm{~L} / \mathrm{min}$

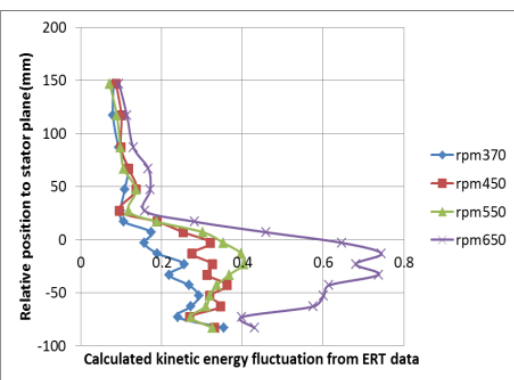

(b-1) ERT result, air rate $80 \mathrm{~L} / \mathrm{min}$

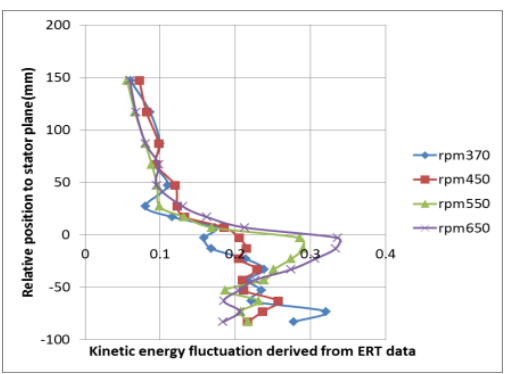

(c-1) ERT result , air rate $100 \mathrm{~L} / \mathrm{min}$ (a-2) PVS result, air rate $40 \mathrm{~L} / \mathrm{min}$

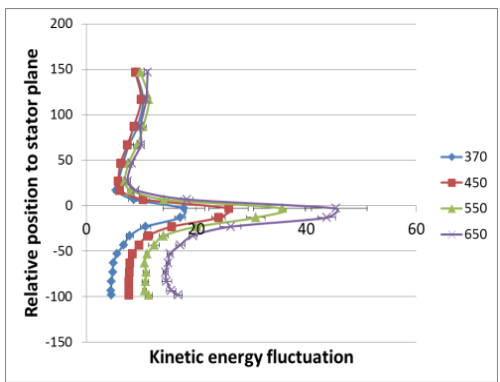

(b-2) PVS result, air rate $80 \mathrm{~L} / \mathrm{min}$

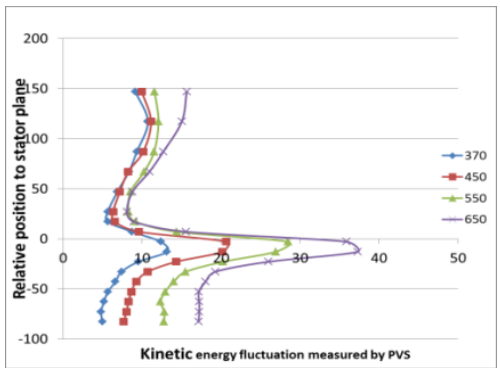

(c -2) PVS result, air rate $100 \mathrm{~L} / \mathrm{min}$ (a-3) PVS VS ERT, air rate $40 \mathrm{~L} / \mathrm{min}$

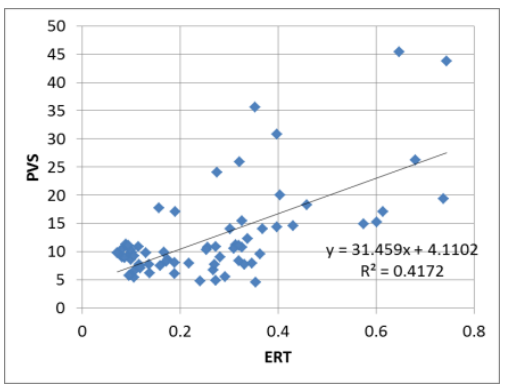

(b-3) PVS VS ERT, air rate $80 \mathrm{~L} / \mathrm{min}$

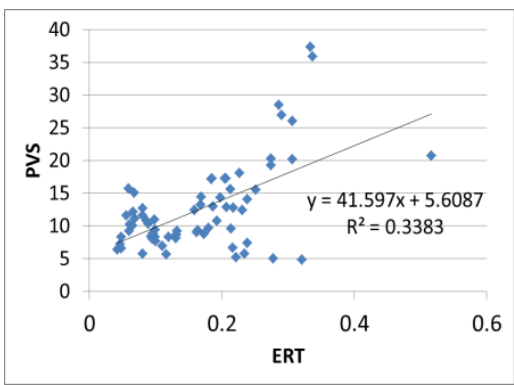

(c-3) PVS vs ERT, air rate $100 \mathrm{~L} / \mathrm{min}$

Figure 8: Comparison of PVS and ERT results at higher air flow rates

\subsection{Comparison of Green-Kubo relation based ERT results with PVS results}

To test the approach based on the Green-Kubo relation described in Section 4.2, the ERT data obtained from measurements performed in the 60 litre flotation cell were processed to calculate the electrical flux $\Phi_{E}$ and the linear transport coefficient $L(0)$ which were then used to derive $F_{e}$. The fluctuation of impulse, $\sigma_{I}$, which is assumed to be proportional to velocity fluctuation, was then calculated to compare with PVS measurement results.

Figure 9 shows the turbulence profiles measured by ERT using the Green-Kubo relation and those measured by the PVS when the flotation cell was operated at a range of different air rates and impeller speeds. The correlations between PVS and ERT measurement are also shown in Figure 9.

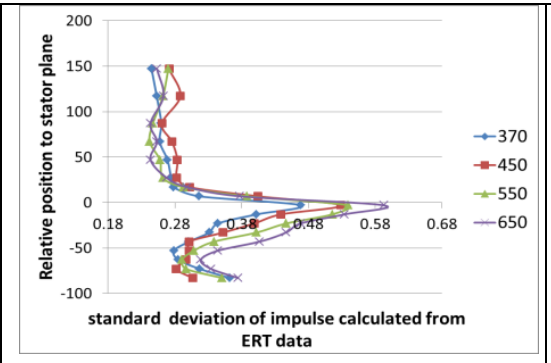

(a-1) ERT result, air rate $40 \mathrm{~L} / \mathrm{min}$

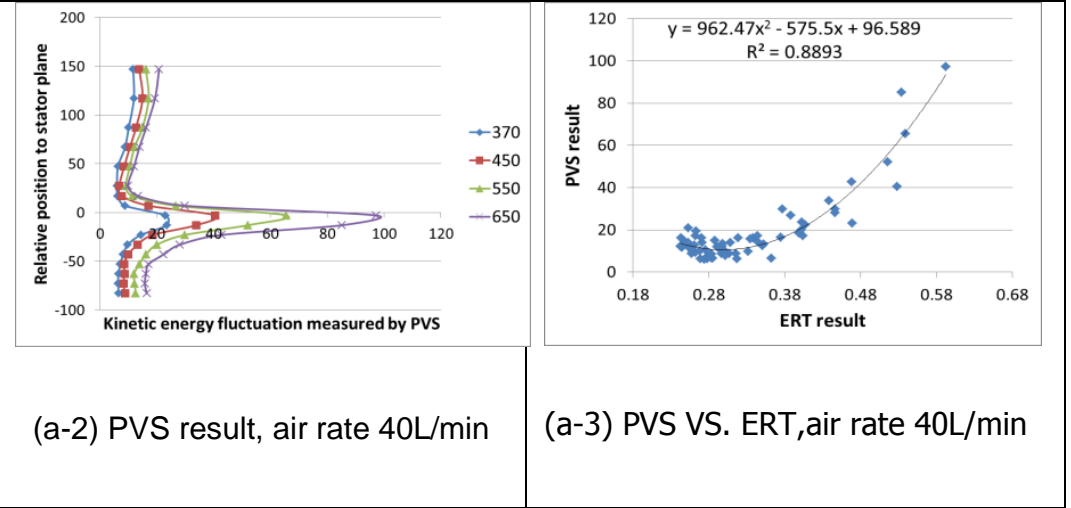




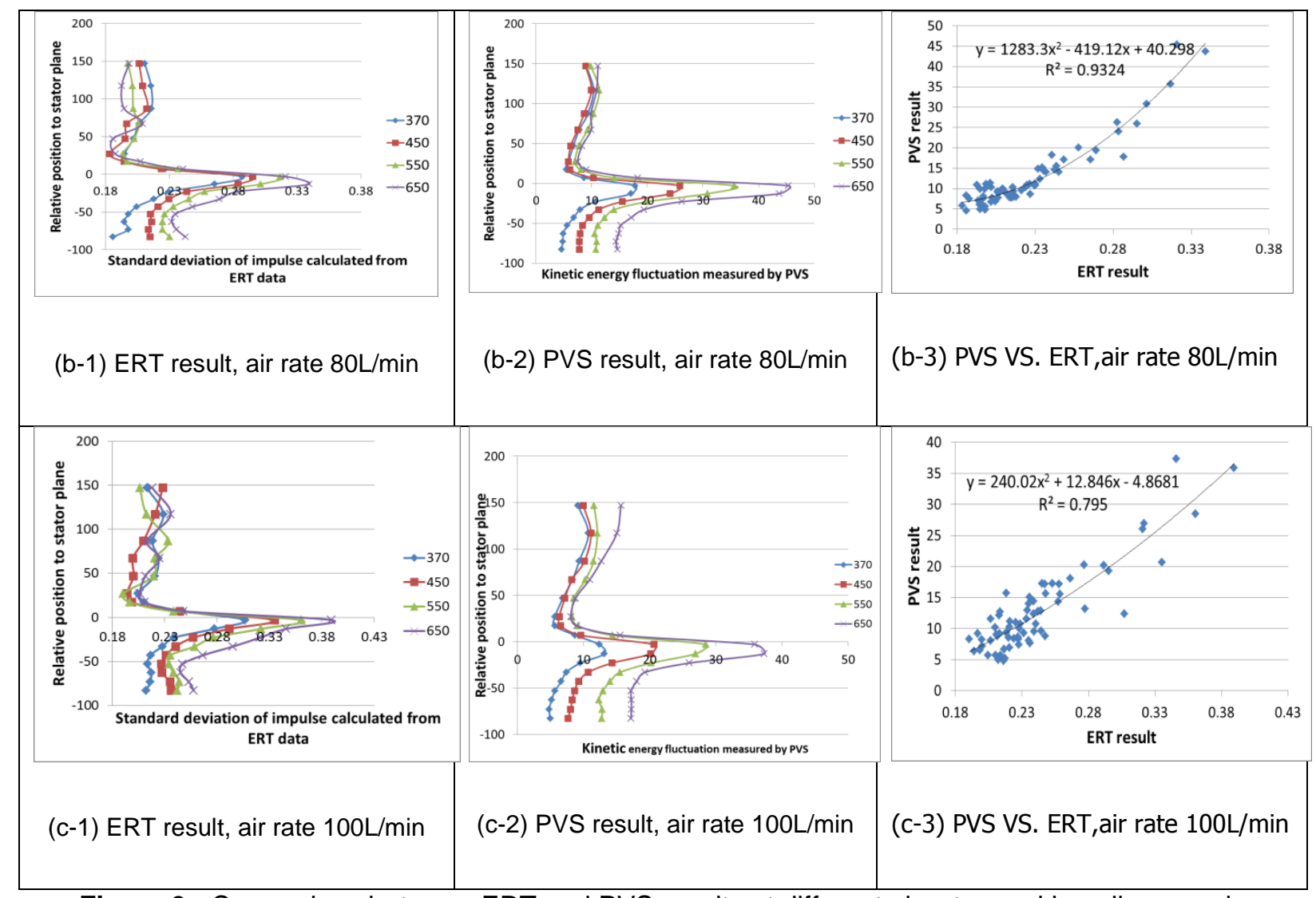

Figure 9: Comparison between ERT and PVS results at different air rates and impeller speeds

It can be seen that the turbulence profiles measured by ERT have the general shape expected in the system - turbulence is low in the upper regions of the cell and increases significantly in the impeller stator region. As impeller speed is increased, the size of the high turbulent zone increases. The ERT turbulence measurement results, however, have a distinctly different shape to the corresponding kinetic energy fluctuation profiles measured by the PVS, with the ERT results extending over a wider distance from the stator plane This is somewhat expected as PVS is measuring kinetic energy fluctuation whereas the ERT measurements are expected to be proportional to velocity fluctuation.

The correlations between ERT results and PVS results at different air rates show that there exists a squared correlation between the $\sigma_{I}$ values and the PVS measured kinetic energy fluctuation values. It has been shown that the kinetic energy fluctuation $\sigma_{v^{2}}$ is proportional to $\sigma_{v}^{2}$ in this flotation cell when operated with water (refer to Equation (9)) and it is being assumed that there is a linear correlation between $\sigma_{I}$ and $\sigma_{v}$. It is therefore expected that $\sigma_{I}$ would exhibit a squared relationship with the PVS kinetic energy fluctuation reading. In Figure 9 (a-3), (b-3) and (c-3), a squared correlation between PVS and ERT data is observed. It should be noted that for Figure 9(a-3) the squared correlation has a minimum value of PVS at ERT value 0.28 , which means that two ERT values at either side the minimum point can correspond to a same PVS value. This is because both the PVS and the ERT measurement data carry errors and this section of the curve is associated with measurements performed in the low turbulence region. When the signal level is low, the error becomes more 
significant and unexpected trends in the correlation may occur. But in general, the squared correlation between PVS and ERT provides strong evidence that the Greens-Kubo relation (outlined in Section 4.2) for processing ERT conductivity data can be used to estimate fluid velocity fluctuation - a measure often used to characterise turbulence intensity. It has been noted from Figure 9 (a-3), (b-3) and (c-3) that the PVS-ERT correlation changes with different air rates and so far no pattern has been identified for it. Therefore more work needs to be done to reveal the underlying mechanisms with varying air rate.

This second data processing approach, in comparison to the variation in conductivity approach outlined above, achieves good correlations over the range of different air rates and impeller speeds tested and depending solely on ERT measurement data. Therefore it is considered the better choice to measure turbulence as it is suitable over a wider range of applications.

\section{Conclusions}

Electrical Resistance Tomography (ERT) is a promising technique that has already been applied to a wide range of process monitoring applications. Since what the ERT measures is conductivity, to develop ERT as an applicable measurement tool for turbulence measurement in flotation cells, data processing methods need to be developed and validated.

In this research, two approaches were proposed to process ERT data. The first involved using the variance of conductivity of the fluid to predict the fluid kinetic energy fluctuation at the measurement position. This approach used measurement data of the piezoelectric sensor and the LDA to establish a model to estimate the kinetic energy fluctuation as a function of impeller speed and position within the cell. The method was tested by performing measurements using ERT in a 60 litre batch flotation cell. The kinetic energy fluctuation calculated from ERT data agreed well with kinetic energy fluctuation measured with the piezoelectric sensor at low air rates.

The second approach involved calculating the fluid impulse fluctuation from ERT measurement data, based on a data processing method using the Green-Kubo relation. This fluid impulse fluctuation, theoretically should be proportional to the velocity fluctuation of the fluid, a turbulence related parameter. Comparison with PVS results provides strong evidence that this is the case. This second data processing approach is considered superior to the first one as the first one requires the development of auxiliary information which would not usually be known for a new system. It also produced more accurate results at higher air rates.

Since turbulence in a flotation cell varies significantly across the cell's cross-section, a turbulence measurement device needs to be able to perform measurements at a series of positions, not just an average over a cross-section. What is therefore required is an intrusive probe instead of the usual application of electrodes fixed to the walls of the cell. In this research, therefore, a probe with 16 electrodes fixed on a small plastic plate was developed, applicable for use not only in laboratory scale cells but also, potentially, in full scale industrial cells. 
Up until now the ERT device has only been applied to measuring turbulence in laboratory scaled cells operating with water and air. Future work will involve testing ERT for measurement of turbulence in three phase systems (water, air and solids) and in industrial scale flotation machines. This work has demonstrated, however, that ERT is a powerful technique that has the potential to be used for turbulence measurement in flotation cells.

\section{Acknowledgements}

Jon Worth of the JKMRC is gratefully acknowledged for his help in setting up the 60 litre flotation cell as well as providing training in its use. Bill Randall of UCT is also thanked for providing the ERT data processing Matlab demo code and for repairing the ERT device. The AMIRA P9P project is also acknowledged for sponsoring and funding this research.

\section{References}

1. Schubert, H. 1999 On the turbulence-controlled microprocesses in flotation machines, International Journal of Mineral Processing, 56(1-4),257-276

2. Fallenius, K. 1987 Turbulence in flotation cells, International Journal of Mineral Processing, 21(1-2),1-23

3. Sherif, S. A. 1998 Hot-wire/film anemometry measurements in flows with heat transfer and signal correction, ISA Transactions, 37(3),141-146

4. Sad Chemloul, N. and Benmedjedi, A. E. K. 2010 Particle velocity detection and measurement in two-phase flow using combined electronic logic system and LDA technique, Flow Measurement and Instrumentation, $21(3), 425-433$

5. Greated, C. A., Skyner, D. J. and Bruce, T. 1992,Particle Image Velocimetry(PIV) In The Costal Engineering Laboratory, 23rd Conference on Coastal Engineering, Venice, Italy, 1992

6. Dickin, F. and Wang, M. 1996 Electrical resistance tomography for process applications, Meas. Sci. Technol., (7),247-260

7. Dines, K. A. and Lytle, R. J. 1979 Computerized geophysical tomography, Proceedings IEEE, 67(7),10651073

8. Lytle, R. J. and Dines, K. A. 1980 Iterative ray tracing between boreholes for underground image reconstruction, IEEE Transactions on Geoscience and Remote sensing, 18,234-240

9. Hua, P. and Woo, J.,1990 Electrical Impedance Tomography, A. Hilger,"Electrical Impedance Tomography",J.G. Webster, Bristol U.K.

10. Wilkinson, A. J., Randall, E. W., Cilliers, J. J., Durrett, D. R., Naidoo, T. and Long, T. 2005 A $1000-$ Measurement Frames/Second ERT Data Capture System With Real-Time Visualization, IEEE Sensors Journal $5(2), 300-307$

11. Dong, F., Xu, C., Zhang, Z. and Ren, S. 2012 Design of Parallel Electrical Resistance Tomography System for Measuring Multiphase Flow, Chinese Journal of Chemical Engineering, 20(2),368-379

12. Wang, M. and Cilliers, J. J. 1999 Detecting non-uniform foam density using electrical resistance tomography, Chemical Engineering Science, 54(5),707-712

13. Cilliers, J. J., Xie, W., Neethling, N. J., Randall, E. W. and Wilkinson, A. J. 2001 Electrical resistance tomography using a bi-directional current pulse technique, Meas. Sci. Technol., 12,997-1001

14. Stevenson, R., Harrison, S. T. L., Miles, N. and Cilliers, J. J. 2006 Examination of swirling flow using electrical resistance tomography, Powder Technology, 162(2),157-165 
15. Harrison, S. T. L., Stevenson, R. and Cilliers, J. J. 2012 Assessing solids concentration homogeneity in Rushton-agitated slurry reactors using electrical resistance tomography (ERT), Chemical Engineering Science, 71(0),392-399

16. Jin, H., Wang, M., Williams, R. A. and Bartolacci, G. 2007 Analysis of bubble behaviors in bubble columns using electrical resistance tomography, Chemical Engineering Journal, 130,179-185

17. Abdullah, B., Dave, C., Nguyen, T.-H., Cooper, C. G. and Adesina, A. A. 2011 Electrical resistance tomography-assisted analysis of dispersed phase hold-up in a gas-inducing mechanically stirred vessel, Chemical Engineering Science, 66(22),5648-5662

18. Kourunen, J., Niitti, T. and Heikkinen, L. M. 2011 Application of three-dimensional electrical resistance tomography to characterize gas holdup distribution in laboratory flotation cell, Minerals Engineering, 24(15),1677-1686

19. Heikkinen, L. M., Kourunen, J., Savolainen, T., Vauhkonen, P. J., Kaipio, J. P. and Vauhkonen, M. 2006 Real Time Three-dimensional Electrical Impedance Tomography Applied in Multiphase Flow Imaging, Meas. Sci. Technol., 17,2083-2087

20. Liter, S. G., Torczynski, J. R., Shollenberger, K. A. and Ceccio, S. L. 2002 Electrical-Impedance Tomography for Opaque Multiphase Flows in Metallic (Electrically-Conducting) Vessels Sandia National Laboratories,Virginia,USA

21. Xie, W., Neethling, S. J. and Cilliers, J. J. 2004 A novel approach for estimating the average bubble size for foams flowing in vertical columns, Chemical Engineering Science, (59),81-86

22. Xie, W., Randall, E. W., Neethling, S. J. and Cilliers, J. J. 2012 An ERT System for Research on Dynamic Foams 6th International Symposium on Process Tomography Cape Town, South Africa, 26 - 28 March 2012

23. Meng, Z., Huang, Z., Wang, B., Ji, H., Li, H. and Yan, Y. 2010 Air-water two-phase flow measurement using a Venturi meter and an electrical resistance tomography sensor, Flow Measurement and Instrumentation, $21,268-276$

24. Lehikoinen, A., Laakkonen, P., Vauhkonen, M., Rinne, A., Saloheimo, K. and Lähteenmäki, S. 2011 Measuring Flotation Process Using Probe Sensor Based on 3D Electrical Resistance Tomography The 5th International Flotation Conference, Cape Town, South Africa, November 14-17, 2011,pp864-869

25. Kourunen, J., Rinne, A., Saloheimo, K., Niitti, T. and Heikkinen, L. M. 2008,Electrical tomography imaging of flotation process in a mechanical flotation cell, 5th International Symposium on Process Tomography, Zakopane, Poland, August 25-26, 2008

26. Kourunen, J., Niitti, T. and Heikkinen, L. M. 2011 Application of three-dimensional electrical resistance tomography to characterize gas holdup distribution in laboratory flotation cell, Minerals Engineering, 24,16771686

27. Bolton, G. T. and Primrose, K. M. 2005 An overview of electrical tomographic measurements in pharmaceutical and related application areas, AAPS PharmSciTech, 6(2),E137-E143

28. Polydorides, N. 2002, Image reconstruction algorithms for soft-field tomography, PhD. Thesis, Institute of Science and Technology,University of Manchester

29. Meng, J., Xie, W., Brennan, M., Runge, K. and Bradshaw, D. 2014 Measuring turbulence in a flotation cell using the piezoelectric sensor, Minerals Engineering, 66-68,84-93

30. Tabosa, E., Runge, K. and Holtham, P. 2012,Development and application of a technique for evaluating turbulence in a flotation cell, XXVI International Mineral Processing Congress (IMPC2012), New Delhi, India, 24-28 September, 2012,pp5377-5390

31. Mathieu, J. and Scott, J. 2000 An introduction to turbulent flow, Cambridge University Press,New York

32. Weaire, D. and Hutzler, S. 1999 The physics of foams, Oxford University Press,New York 
33. Fornasini, P. 2008 The uncertainty in physical measurements-An introduction to data analysis in physics library, Springer, Italy

34. Nissinen, A. 2011, Modelling errors in electrical impedance tomography, PhD., Department of Applied Physics,University of Eastern Finland

35. Brennan, M. 2013 Measurement by LDA and modelling by CFD of flotation cell hydrodynamics,Julius Kruttschnitt Mineral Research Centre,University of Queensland, Australia

36. Green, M. S. 1954 Markoff Random Processes and the Statistical Mechanics of Time - Dependent Phenomena. II. Irreversible Processes in Fluids, The Journal of Chemical Physics, 22(3),398-413

37. Kubo, R. 1957 Statistical-Mechanical Theory of Irreversible Processes. I. General Theory and Simple Applications to Magnetic and Conduction Problems, Journal of the Physical Society of Japan, 12(6),570-586 